\title{
Risk Reduction Potential of REACH Authorisation
}

\author{
Romy MARX* and Aart ROUW
}

\section{INTRODUCTION}

\section{Scope}

An important objective of authorisation in the REACH Regulation (EC/1907/2006) ${ }^{1}$ is to encourage substances of very high concern (SVHCs) to be eventually replaced by less hazardous alternatives, and to reduce risks as long as SVHCs are still used.

The group of Applications for Authorisations (AfAs) for the use of chromium trioxide $\left(\mathrm{CrO}_{3}\right.$, a Carc 1A substance) in chrome plating is now being discussed union-wide and a major part of these AfAs and the resulting opinions of the Risk Assessment Committee (RAC) of ECHA are now available in the public domain.

This allows a comparison of the submitted information to be made, and the reported status to be judged regarding alternatives and risk reduction strategies. It also provides a good view on the potential of REACH authorisation to reach its objectives. In analysing the AfAs for $\mathrm{CrO}_{3}$ submitted so far, this report will illustrate why substitution perspectives seem less strong in reality than they were supposed to be. In these AfAs improvements for risk reduction, especially regarding specific workplace measures, have been described. But these measures can still be improved further and will need to be reassessed after the first period of authorisation.

The necessity and usefulness, and even the legality, of identifying $\mathrm{CrO}_{3}$ as a SVHC and making it subject to the REACH authorisation regime has been intensively debated at national levels. The inclusion into Annex XIV without an exemption being granted for chrome plating was even brought before the European Court of Justice. ${ }^{2}$

The ECJ rejected the appeal of VECCO (a consortium of German companies, active in chrome plating) and said that the subsequent inclusion of $\mathrm{CrO}_{3}$ into Annex XIV after the initial inclusion in the candidate list was according to the law. The judgment concludes that measures under the Chemical Agents Directive $(\mathrm{CAD})^{3}$ and Carcinogens and Mutagens Directive (CMD) ${ }^{4}$ by themselves do not constitute sufficient reason for

\footnotetext{
* Federal Institute for Occupational Safety and Health, Friedrich Henkelweg 1-25 44149 Dortmund, Germany. Romy Marx and Aart Rouw confirm that there are no known conflicts of interest associated with this publication and that there has been no financial support for this work that could have influenced its outcome.

1 Regulation 1907/2006/EC on the Registration, Evaluation, Authorisation and Restriction of Chemicals (2016).

2 Judgment of 25 September 2015, T-360/13, VECCO and Others v Commission, EU:T:2015:695.

3 Directive 98/24/EC on the protection of the health and safety of workers from the risks related to chemical agents at work (1998).

4 Directive 2004/37/EC on carcinogens und mutagens at work (2004).
} 
granting exemptions of authorisation duties under REACH. Both CAD and CMD are not substance specific and do not press for substitution in the same way as the REACH regulation. This was confirmed by the ECJ ${ }^{5}$ after VECCO objected to the first decision.

\section{Assessment of risks}

$\mathrm{Cr}^{\mathrm{VI}}$ compounds are carcinogens without a threshold. This means that even the slightest exposure carries a risk and that there is no "safe" exposure. Therefore, for substances like $\mathrm{CrO}_{3}$ the relationship between exposure and risk (ie how many workers develop cancer out of a collective) needs to be determined. This allows the calculation of how many cancer cases will (statistically) occur in the facility of the applicant under the reported exposure.

However, deriving such an exposure-risk relationship is complicated. In the case of $\mathrm{Cr}^{\mathrm{VI}}$, the development of lung cancer has been shown to be correlated to exposure to $\mathrm{Cr}^{\mathrm{VI}}$. However, different analyses have led to different relationships. ${ }^{6}$ This may cause lengthy debates during the authorisation process. RAC prepared an analysis of available data $^{7}$ for $\mathrm{Cr}^{\mathrm{VI}}$ compounds in order to create a common reference set of values.

According to $\mathrm{Cr}^{\mathrm{VI}}$ reference values, the risk of developing lung cancer at an exposure level of $1 \mu \mathrm{g} / \mathrm{m}^{3}$ for 8 hours/day over a 40 -year period (ie a total working life) amounts to 4:1,000. This also corresponds to the risk level derived in Germany. ${ }^{8}$ Small companies with a few dozen workers will therefore hardly ever find cases in their company history. This argument was often brought into the discussion by such companies and their VECCO representatives. They took this as an indication that apparently there was no problem in using $\mathrm{Cr}^{\mathrm{VI}}$.

In various uses of $\mathrm{Cr}^{\mathrm{VI}}$ substances, a wide range of exposure values (and therefore related risks) has been reported. In this respect, it is important that REACH does not use a maximum level of risk, above which authorisation will automatically be rejected. In the absence of feasible alternatives, presenting a socio-economic analysis is the only option available to the applicant to obtain an authorisation. This may cause problems with national regulations, which may use fixed exposure limits or set a maximum risk level. If benefits outweigh the risks, an authorisation may be granted, whereas a national regulation may still oblige a company to apply additional risk control measures.

\section{Analysed data (status $1^{\text {ST }}$ Quarter 2017)}

The analysis concentrates on the use of $\mathrm{CrO}_{3}$ in Functional Chrome Plating (FCP).

The primary analysis focused on workplace risk reduction measures as already laid down in worker legislation. One AfA $^{9}$ claims FCP covers about 840-1,590 sites, with 23,000 potentially exposed workers. The uses of $\mathrm{CrO}_{3}$ in FCP lead to products (ie the

5 Judgment of 13 July 2017, C-651/15 P, VECCO and Others v Comission.

6 A Seidler et al, "Systematic review and quantification of respiratory cancer risk for occupational exposure to hexavalent chromium" (2013) 86 International Archives of Occupational and Environmental Health 943.

7 ECHA, Application For Authorisation: Establishing A Reference Dose Response Relationship For Carcinogenicity Of Hexavalent Chromium (2013).

8 AGS, Begründung zu Chrom VI in TRGS 910 (2014).

9 ECHA, Socio-economic Analysis of Application No 32-02 (2016), <echa.europa.eu/documents/10162/f33c6bb09d53-4a43-9061-08efa8026724>, visited 4 January 2018. 
metal or plastic article being chrome-plated) that contain metallic chromium, which is not hazardous. In other uses (not analysed here) this is different (eg chromates used for passivation).

The essential parts of each AfA (RAC and SEAC opinions, the analysis of alternatives and the chemical safety reports describing the risk assessment) are available from the ECHA website. ${ }^{10}$ This gives an overview on the various uses, risk reduction measures and alternatives reported by the applicants. Up to March 2017, 49 AfAs for different uses of $\mathrm{CrO}_{3}$ have been submitted. Of these, 22 cover FCP and formed the basis of our analysis.

Some AfAs may describe different variations of the plating process. This may lead to different risk management measures (RMM) and exposure levels. Therefore, such variations were counted separately, resulting in 26 cases available for analysis. Moreover, the scope was narrowed to those parts of the process (so called "worker contributing scenarios" (WCS)) that describe the actual plating process. In this process step aerosol formation may occur, which is assumed to contribute most to worker risk. In order to understand the risk reduction potential of Authorisation under REACH it is necessary to pick up a specific example from a larger data pool to be able to draw general conclusions.

\section{Findings from AfAs for the use of $\mathrm{CRO}_{3}$ IN FCP}

\section{Alternatives}

We found that the use of $\mathrm{Cr}^{\mathrm{III}}$ electrolytes in an electro-deposition bath (either as a direct replacement of $\mathrm{Cr}^{\mathrm{VI}}$ or in combination with other treatments) is mentioned in 19 out of 22 AfAs as the most promising alternative. In this case, the deposition of non-toxic chromium metal is, at first sight, chemically identical to the result from a hexavalent chromium bath. Moreover, basically the same equipment can be used. However, AfAs discussing $\mathrm{Cr}^{\mathrm{III}}$ agree that, although plating with $\mathrm{Cr}^{\mathrm{III}}$ is possible and already in commercial use in some non-critical areas, the micromorphology of the resulting layer is different and the properties often inferior compared to $\mathrm{Cr}^{\mathrm{VI}}$. Moreover, $\mathrm{Cr}^{\mathrm{III}}$ bath equipment needs to be more sophisticated and to get a stable process monitoring of bath properties is more critical. Nevertheless, applicants expect improvements in this technology in the future. All other cited alternatives (eg nitrocarburation, plasma treatments, various combinations of nickel and $\mathrm{Cr}^{\mathrm{III}}$ deposition) do not match the properties of chromium (optics, hardness, adhesion low friction, corrosion protection). Therefore, such "alternatives" may find use in less critical applications. However, most of hard chrome plating is done in small companies whose unique competitive advantage is flexibility and the ability to serve different customer demands. Most do not have the option to run multiple technologies side by side. Therefore, they need continued use of $\mathrm{Cr}^{\mathrm{VI}}$ to stay in business.

\section{Technical risk management measures}

The question whether risks have been reduced because of authorisation is difficult to answer, because there is no overview of how the situation was before authorisation.

10 Only freely-accessible documents were used, in order to avoid confidentiality issues. 
Table 1. Short version of the example for FCP of TRGS 460

\begin{tabular}{|c|c|c|c|}
\hline Process & $\begin{array}{l}\mu g C r^{V I} / m^{3} \\
\left(95^{\text {th }} \text { percentile }\right)\end{array}$ & Technical measures & Rating \\
\hline A & up to $24.6^{*}$ & rim ventilation & industrial practice \\
\hline B & up to $12^{\#}$ & A + wetting agent (or floating bodies) & industrial practice \\
\hline $\mathrm{C}$ & up to $8.4^{\#}$ & $\mathrm{~B}+$ air-conditioning systems & \\
\hline $\mathrm{D}$ & up to $6-8$ & A + covering of bath & \\
\hline $\mathrm{E}$ & up to $4.6^{*}$ & $\mathrm{~B}+$ covering of bath & \\
\hline $\mathrm{F}$ & up to $3.2^{\#}$ & $\mathrm{C}+$ covering of bath & \\
\hline G & $<1 \mu \mathrm{g}^{\#}$ & $\mathrm{E}+$ ventilation booth at filling car & State of the art \\
\hline $\mathrm{H}$ & $<1 \mu \mathrm{g}^{\#}$ & $\mathrm{G}+$ air-conditioning system & State of the art \\
\hline
\end{tabular}

* measured, \# modelled/estimated

Submitted AfAs fall in two categories. There are "upstream applications" by manufacturers, importers or formulators ( 5 out of 22 in our selection) who apply for an authorisation for many uses and then sell the substance to any downstream user needing it. This may serve the majority of the 840-1,590 companies using $\mathrm{CrO}_{3}$. Other applications are submitted by single companies or consortiums of a few companies. From assessing these AfAs it can be concluded that companies have analysed how they operate, what steps their processes include, where the highest exposures (ie the highest risks) can be expected, and what RMMs are already in place or can be easily implemented. In some cases measured exposure values are presented. We would like to stress that the amount of information and data that has become available allows, maybe for the first time, a comparison to be made between operational practices in similar uses throughout Europe, which is a leap forward for the assessment of occupational safety.

The economic situation of companies (and what they can invest in additional occupational safety measures) may differ. However, in the future it will be possible to better compare similar companies, application fields or uses in different countries.

In Germany, the implementation of a national risk relation value and $\mathrm{CrO}_{3}$ authorisation resulted in a description of a "state of the art" example for FCP (technical rule for hazardous substances (TRGS) 460). ${ }^{11}$ Technical measures are described and are related to $\mathrm{Cr}^{\mathrm{VI}}$ exposure values that may be expected. In cases of reinvestment or renovation of equipment, this comparison may allow informed decisions to adapt the best available technology (eg replacing a bath with only local exhaust ventilation with a closed system, see Table 1). This will not remove all differences between operations in different plants, but for companies lacking modern standards it will be difficult to argue that improvements are technically not possible. The derivation of such a "state of the art" is in principle possible from the information given in AfAs, also for other substances.

The AfAs give insights into which measures are used in the FCP industry, and allow conclusions to be drawn regarding related exposure. In reality this is less straightforward because the upstream applications cover a large number of downstream users, at the expense of a detailed description of measures and exposure at individual companies.

11 AGS, Praxisbeispiele zur TRGS 460 “Handlungsempfehlungen zur Ermittlung des Standes der Technik” (2015). 


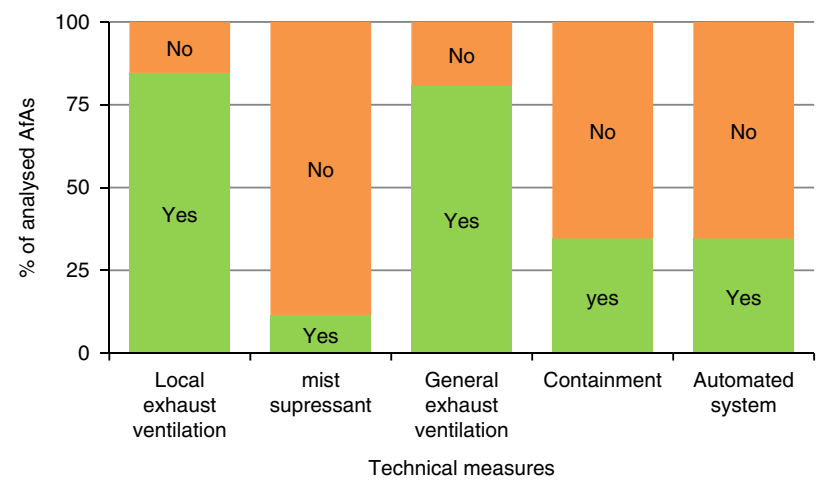

Figure 1. (Colour online) Reported usage of technical measures in percent of the 26 analysed WCS

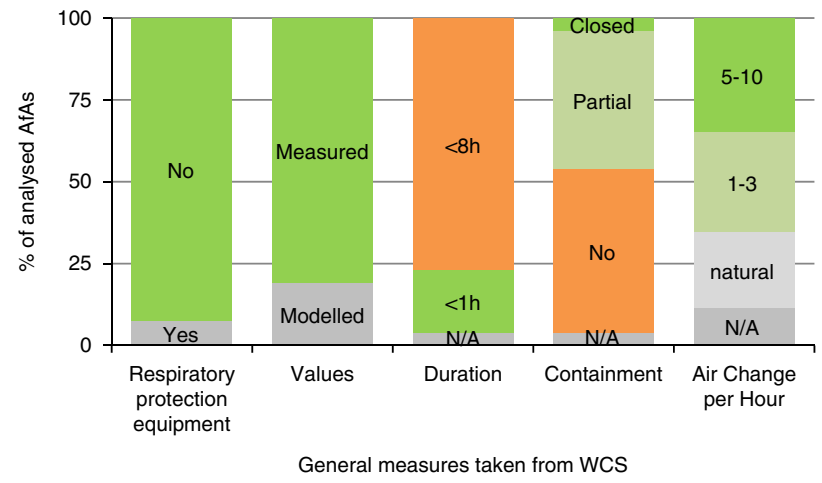

Figure 2. (Colour online) General measures given in the WCS $(\mathrm{N}=26)$ of the plating process

Moreover, in many applications, exposure data are based on modelling, not on measurements. Also, it is not always clear how these relate to individual work conditions. The descriptions, especially in upstream applications, leave room for improvement. These shortcomings have also been identified by RAC, as shown in the opinion documents.

Despite these uncertainties the results regarding some parameters are shown in Figures 1 and 2. This is based on the 26 WCS describing the plating step, as defined in section II.

Figure 1 illustrates the technical measures that should in principle be regarded and the percentage of WCS that actually mention their use. The results show a very common use of ventilation at the bath itself or at the workplace in general. However, surfactants which are often discussed in the AfA as a technical means of reducing aerosol formation are only described in a few WCS. This seems surprisingly low. We cannot exclude that many applicants did not specifically include this RMM. Either they considered this as standard anyway, or they did not specifically take this into account in their modelling calculations. Regarding bath coverage, it should be considered that details of the process can prevent a practical implementation. Therefore, the finding of "only" a quarter of applications using bath enclosure is not surprising. Most existing FCP plants have a low 
level of automation. This is different for more modern plants. The findings regarding the scope/nature of technical measures displayed in the AfAs are in line with industrial standards in FCP plants, at least in Germany.

Analysing the WCS for the plating process, Figure 2 summarises some interesting facts. Only a low number of applications report use of respiratory protection equipment. Additionally, most exposure values were obtained by personal measurements, not by modelling. Another positive finding is the fact that more than half of the applications include good (1-3 air changes per hour) to very good (5-10 air changes per hour) ventilation rates.

However, the descriptions of duration of the work and of bath containment show possibilities for improvements. At least $25 \%$ of the WCS provide concrete indications of the duration for a specific worker task (eg three minutes for dipping and immersion), whereas $75 \%$ only gave the maximum amount of time spent at the workplace (ie $<8 \mathrm{~h}$ ). Here, there is room for differentiation in the description, leading to a more precise knowledge of the actual exposure time during the critical task. The same applies for the level of containment. Often it does not become clear what the actual level of containment is, and more than half of the applications had no coverage of the bath at all (which, if present, will give maximum exposure reduction according to the state of the art description).

These findings can only be used to judge the $\mathrm{CrO}_{3}$ plating process. Weighing or maintenance (usually also attributed with high potential for exposure) are associated with different risks which would lead to different measures.

\section{REFLECTION ON FINDINGS AND CONCLUDING REMARKS}

At the introduction of REACH, it was not easy to predict how authorisation of chemicals would work in practice. Considerable experience has now been collected in the preparation, submittal, and discussion of AfAs. This experience originates from discussions between the legislators and industrial representatives (individual companies as well as consortiums) on national and union-wide levels, including ECHA and commission representatives. There is material in the public domain that gives the legislator indications if and how this new concept contributes to a risk reduction in the handling of hazardous chemicals. This information and corresponding interpretation is not summarised in any report yet (a report commissioned by the EU-Commission for the REACh-Review was to be finalised in September 2017), but was gathered by the authors by studying and analysing AfAs and RAC/SEAC opinion (various documents) on ECHAs website. The interesting question at the final part of this article is to judge whether $\mathrm{REACH}$ authorisation is really living up to its objectives.

\section{Substitution}

Our approach cannot identify companies that went out of business or which were able to find alternatives for their specific uses and therefore do not need an authorisation. In addition, it cannot be ruled out, that the use (and the risks) have been relocated to 
companies outside the EU - a scenario which is often used by companies as a potential threat.

However, it is our impression that, at least within Germany, the vast majority of plating companies will use an authorisation, either by an application of their own or via an upstream application of their supplier. Most companies seem to have accepted that an authorisation for the use of $\mathrm{Cr}^{\mathrm{VI}}$ will be part of future business reality.

The benefit of authorisation is that potential substitution possibilities have now been described in much more detail and transparency than ever before. In the case of $\mathrm{Cr}^{\mathrm{VI}}$ in less demanding applications, this may lead to substitution within the next 10 years. For FCP in high performance applications, which we chose as example of choice, mid-term perspectives for substitution do not look very promising. Authorisations for most such uses may survive well beyond the first period of authorisations.

Overall, we are of the opinion that not only FCP is limited in alternatives. In general it might be assumed that companies will only apply for an authorisation if no alternative is available. Therefore the proposed substitution pressure of REACH authorisation will only be effective in use areas where alternatives have been under development prior to REACH.

\section{Safe use}

AfAs seem to have increased attention for occupational safety and have led to a general awareness amongst employers of monitor and document safety standards. It remains to be seen if this was a one-off exercise, or if the stress of preparing for a review of authorisations will cause continuous improvement. Evaluation of review reports by RAC and SEAC submitted after the first authorisation period has expired will be crucial. As section III. 2 shows, applicants address the measures that are generally possible in the chrome plating industry. But sometimes they fail to include the detailed description of these measures in the WCS. This makes it difficult to conclude whether all possible measures have been implemented and whether a particular RMM is appropriate and effective in limiting the risk. We regard the description of workplace measures as maybe the best possibility to judge and compare AfAs in a transparent way. Collection of relevant data may lead to a European-wide overview of what constitutes best practice in the field of FCP, both in technical and organisational measures. Ideally, it may be imagined that the assessing committees will make use of this information and define a "state of the art" for the sector. Implementation of specific technical measures may then be made a prerequisite condition during future reviews of AfAs. In that, information drawn from AfAs would eventually lead to a risk reduction in the use of $\mathrm{CrO}_{3}$.

We think that the general idea of analysing AfAs can be applied to any other SVHCs also and we are convinced that deriving best practices for the workplace can be a very effective tool to reduce occupational risks. Worker legislation demands this in principle already and REACH authorisation might be a helpful addition in improving workplace safety for substances without a threshold.

Generally, the presentation of data in AfAs was acceptable (although more pictures would help) with room for improvement in the level of detail. It has to be noted that in this first round of AfAs neither industry nor committees had a good overview of the details that would be needed. All parties now have a better (but not final) view on what 
should be included. The next review period(s) will show whether applications improve. Ideally, to pave the way for a community-wide development towards improved harmonisation in technical and organisational standards, committees need a precise picture of those uses for which authorisation is sought and of the best available techniques to minimise risks. Only then can a process develop in which companies with safer use can be identified and those that do not manage to achieve high standards will be superseded.

Another important step forward will be to support upstream AfAs. This will reduce workload for the committees and simplify the system. This option is explicitly laid down in the REACH legal text. So far, the upstream AfAs lack the necessary level of detail to judge evenly every covered use. We strongly recommend supporting consortiums in improving their upstream applications, and finding ways to supply the level of detail without making the AfAs themselves excessively long.

\section{Concluding remarks}

Use of $\mathrm{CrO}_{3}$ is no longer allowed after the sunset date (21 September 2017) unless an AfA has been submitted. The published AfAs show a rather sobering view on alternatives and reveal the need to refine description of use scenarios. On the positive side, alternatives, as well as exposure values and risk assessment were publicly discussed. The needed improvements in these areas should be in focus after the first review period has expired. We conclude that authorisation under REACH is still in its infancy and industry and regulators are still on a learning curve. The way AfAs have been designed and information presented allows the legislator to have a precise view of the actual and the possible workplace conditions in the use of $\mathrm{CrO}_{3}$ in FCP.

It is still too early to give a final judgment on the impact of authorisation on the safety of the worker. This is also true for other substances subject to authorisation. Through data analysis we have outlined one way to improve worker safety, in defining best practices which are tied to the granting of authorisations. This applies not only to $\mathrm{CrO}_{3}$ but to any other SVHCs under the authorisation regime. In our opinion, the authorisation under REACH has the potential to greatly reduce risks at the workplace. It is likely that this will not happen due to a direct phase out and replacement by alternatives, but rather due to definition of workplace measures that allow for the safest handling. 